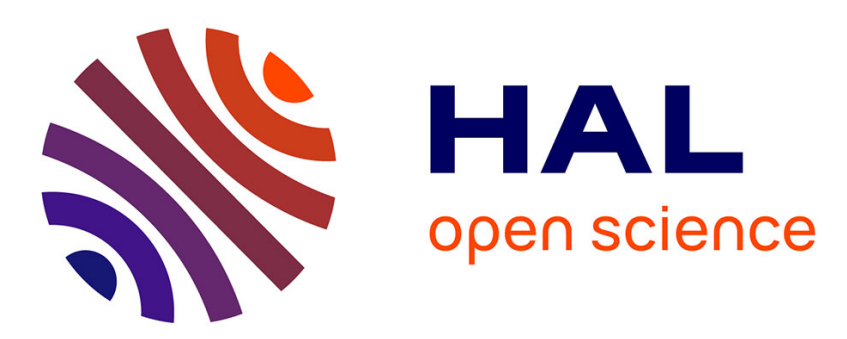

\title{
Ecuries et autres bâtiments fonctionnels dans le château d'Urvei (Sardaigne) aux XIIIe et XIVe siècles
}

\author{
Jean-Michel Poisson
}

\section{To cite this version:}

Jean-Michel Poisson. Ecuries et autres bâtiments fonctionnels dans le château d'Urvei (Sardaigne) aux XIIIe et XIVe siècles. 2013. halshs-01023383

\section{HAL Id: halshs-01023383 \\ https://shs.hal.science/halshs-01023383}

Preprint submitted on 12 Jul 2014

HAL is a multi-disciplinary open access archive for the deposit and dissemination of scientific research documents, whether they are published or not. The documents may come from teaching and research institutions in France or abroad, or from public or private research centers.
L'archive ouverte pluridisciplinaire HAL, est destinée au dépôt et à la diffusion de documents scientifiques de niveau recherche, publiés ou non, émanant des établissements d'enseignement et de recherche français ou étrangers, des laboratoires publics ou privés. 


\title{
ECURIES ET AUTRES BATIMENTS FONCTIONNELS \\ DANS LE CHATEAU D'URVEI (SARDAIGNE) AUX XIII ${ }^{\mathrm{e}}$ ET XIV ${ }^{\mathrm{e}}$ SIECLES
}

\author{
JEAN-MICHEL POISSON*
}

Le castrum d'Urvei a fait l'objet de fouilles archéologiques entre 1990 et 1994 par le CIHAM (Lyon) en collaboration avec l'Ecole française de Rome et l'Université de Cagliari' Il est situé dans la Sardaigne centro-septentrionale, sur la commune d'Ozieri, province de Sassari, à quelques kilomètres à l'ouest de Tula. C'est un site de hauteur, installé sur la côtière, orientée nord-est-sud-ouest, qui borde au sud une zone de plateaux élevés, le Monte Sassu, et domine, à $640 \mathrm{~m}$ d'altitude, la plaine d'Ozieri. L'établissement lui-même est installé sur un plateau allongé, situé en bordure d'une falaise d'environ $300 \mathrm{~m}$ de haut : le choix de protection naturelle et de domination d'un vaste espace est ici manifeste. Ce site est implanté dans un secteur qui constitue, au Moyen Âge, une zone frontalière entre deux royaumes, Gallure et Torres, à l'époque des « judicats », États autochtones indépendants (jusqu'à la fin $\mathrm{du} \mathrm{XII}^{\mathrm{e}}$ s.), d'une part, mais aussi d'autre part sur la limite entre deux aires d'influence distinctes : une zone sud sous contrôle pisan et une zone nord sous contrôle génois, au XIII ${ }^{\mathrm{e}}$ et au début du XIV ${ }^{\mathrm{e}}$ siècle (Cf. carte). Ce site médiéval déserté avait déjà attiré l'attention de plusieurs historiens, et son emplacement précis avait été repéré à l'occasion d'une campagne de prospections archéologiques portant sur les habitats fortifiés médiévaux de Sardaigne ${ }^{2}$.

Urvei apparaît dans la documentation écrite au milieu du $\mathrm{XI}^{\mathrm{e}}$ siècle : il apparaît alors comme un domaine rural détenu par une famille de possédants qui en porte le nom, et qui se manifestent à trois reprises dans la documentation : Therkis d'Orbei et son frère Egithu, vers 1064, Comita de Orbei vers 1070-80. Ces maiores locaux semblent occuper une position économique et sociale importante dans le secteur, puisqu'on les voit disputer à l'évêque de Bisarcio la possession de serfs, et intervenir dans des actes publics accompagnés de leur garde personnelle ${ }^{3}$. On ne sait rien du site lui-même à cette époque, mais le choix d'un emplacement remarquable topographiquement suggère une position dominante, protégée, renforcée par l'existence d'un groupe armé, la garde. Cependant le lieu ne semble pas à proprement parler fortifié à cette époque, même si son emplacement est naturellement bien défendu ${ }^{4}$. Dans la première moitié du XIII ${ }^{\mathrm{e}}$ siècle, au moment où les Pisans mettent la main sur le judicat (royaume) de Gallure, les nouveaux maîtres détiennent un château édifié sur le site, mentionné dans le traité pisano-génois de 1288, et dans lequel il est logique de penser qu'ils ont installé une garnison destinée à assurer le contrôle de cette zone de frontière avec les possessions génoises. L'initiative de l'érection du château lui-même semble devoir revenir à

\footnotetext{
* Ecole des Hautes Etudes en Sciences Sociales, UMR 5648 - CIHAM (Lyon).

Avec la collaboration, pour les dessins, de C. de Mauroy, K. Mercier, J. Planchon, V. Serdon et D. Séris.

${ }^{1}$ POISSON 1991 et 1992.

2 AMADU 1963 ; DAY1973, p. 109-110; AMADU 1975 et 1984 ; MELONI 198819911992 et 1994. POISSON 1986 et 1990.

${ }^{3}$ [v. 1064] deux fiches du condaghe (cartulaire) du prieuré de S. Pietro de Silki portent sur un litige pour la possession de quatre serfs fugitifs entre Giorgio Maiule évêque de Bisarcio et les deux frères (BONAZZI 1900, $\mathrm{n}^{\circ} 42$ et 44); [v. 1070-80] une fiche du condaghe de l'évéché de Bisarcio mentionne comme témoin d'une acquisition faite par l'évêque Nicodemo : Comita de Orbei cum tota iscolca sua (TOLA 1861, XIe s. n²13). A noter qu'au même endroit, en premier dans la liste des témoins (et juste avant Comita) figure l'évêque luimême: Ego piscopu Nichodemu cum tota iscolca mea. Sur la signification de iscolca ou scolca (garde personnelle), Cf. LA CORTE 1937.

${ }^{4}$ Le secteur du Monte Sassu est densément occupé dès l'époque du Bronze final, et plusieurs structures mégalithiques (nuraghes) sont présentes dans le secteur, notamment dans les parages du site médiéval d'Urvei. Cf. AMADU 1978.
} 
l'allié des Pisans dans la guerre de conquête du judicat de Torres (Logudoro), le juge d'Arborea Mariano II (1241-1291) qui, après s'être peu à peu affranchi d'une tutelle pisane trop étroite, maintint une communauté d'intérêts avec eux contre les Génois. Le traité de paix de 1288, consécutif à la bataille de la Meloria qui marque la fin de la suprématie pisane en Tyrrhénienne, oblige la Commune de Pise, en Sardaigne, à se défaire en faveur de Gênes, en particulier, de quatre châteaux édifiés en Logudoro, parmi lesquels castrum quod vocatur

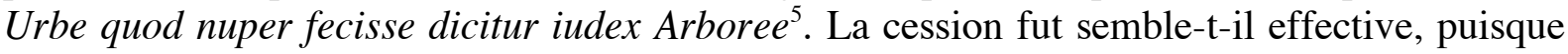
le texte d'une trêve de vingt-cinq ans signée en 1299 entre Pise et Gênes, qui prévoyait le paiement d'une forte somme à cause de la non restitution des châteaux prévue au traité de 1288, ne mentionne plus celui d'Urvei'.

Par la suite, on ignore si le château reste en mains génoises. Au cours des tractations, vers 1304-1308, entre la dynastie génoise des Doria et Jacques II d'Aragon qui cherche des alliés en vue de la conquête de la Sardaigne, il n'est pas question d'Urvei. Cependant les Doria, qui étaient implantés depuis de XII ${ }^{\mathrm{e}}$ siècle dans la partie centrale du Logudoro, obtiennent de se faire attribuer pour prix de leur alliance les châteaux voisins de Monteacuto, de Goceano et de Montiferru, qui étaient déjà les trois châteaux cédés, avec Urvei, à Gênes par Pise en $1288^{7}$. Cela signifie qu'alors Urvei est soit resté aux mains du juge d'Arborea dès la fin du XIII ${ }^{\mathrm{e}}$ siècle (la région du Monteacuto sera en effet constituée en apanage pour son fils Giovanni), soit est en ruines et peut-être abandonné à cette époque.

Dans les premières années de la domination aragonaise (deuxième quart du $\mathrm{XIV}^{\mathrm{e}} \mathrm{s}$.), qui marque la fin de la prépondérance pisane dans l'île, les Doria, cherchent alors à se maintenir dans leurs domaines du Logudoro et, en occupant le château d'Urvei et en le restaurant, tentent d'y organiser une résistance militaire à la Couronne d'Aragon. Deux actes nous informent de cette situation en 1331 : le juge d'Arborea Ugone informe le roi d'Aragon que Niccolò Doria s'apprête à fortifier le Mons de $\operatorname{Orbei}^{8}$. Ces travaux ne sont pas connus précisément, mais ils consistèrent certainement à s'installer dans l'ancien château d'Urvei et à le remettre en état. En même temps, ils édifient ex novo une forteresse près de Giave, Rochafort (aujourd'hui Pianu Roccaforte), à $35 \mathrm{Km}$ au sud sud-ouest d'Urvei. Ces opérations sont mentionnées dans sept lettres envoyées entre le 8 février 1331 et le 20 février 1332 au roi d'Aragon par ses alliés sardes, qui le pressent d'interdire ces constructions et même de les faire détruire'. Après cela, le château d'Urvei n'est plus mentionné dans les textes, et il est fort probable qu'il ait été attaqué par l'armée aragonaise, pendant ou après la restauration engagée par les Doria, et détruit pour éviter toute velléité de résistance, ce qui a du se produire vraisemblablement dans les années 1330. On sait en effet que les troupes du gouverneur aragonais Ramon de Cardona ravagent la région à ce moment ${ }^{10}$.

Les textes sont encore plus rares concernant l'existence à Urvei d'un habitat. Pour le $\mathrm{XI}^{\mathrm{e}}$ siècle, l'existence d'une famille portant ce nom de lieu n'implique pas qu'il y ait pu y avoir, sur place, autre chose qu'un centre domanial comprenant peut-être une résidence et des bâtiments destinés à l'habitation des serfs. Pour le XIII ${ }^{\mathrm{e}}$ siècle, l'existence d'un château n'implique pas non plus qu'il y ait eu sur place autre chose qu'un ensemble fortifié destiné à abriter une garnison et du personnel de service. C'est l'existence d'une église qui atteste la présence d'une communauté d'habitants, à partir du XIV siècle : les Rationes decimarum font

\footnotetext{
${ }^{5}$ Même formulation dans l'approbation par la commune de Pise des chapitres de la paix future, le 3 avril (TOLA 1861, XIIIe s., $\left.n^{\circ} 125\right)$ et dans le traité lui-même, du 15 avril $\left(n^{\circ} 127\right)$. L'identification se base à la fois sur la parenté Urbe - Urvei et sur la proximité des trois autres châteaux : Mons Cutianus (Goceano), Mons de Verro (Montiferru) et Mons Acutus (Monteacuto). Cf. MELONI 1988.

${ }^{6}$ TOLA 1861 , XIIIe s., $n^{\circ} 141$.

${ }^{7}$ SALAVERT Y ROCA 1956, $\mathrm{n}^{\circ} 182,249,258,270$.

${ }^{8} 17$ février (CASULA $\left.1970, \mathrm{n}^{\circ} 112\right)$ et 11 mars $\left(\mathrm{n}^{\circ} 130\right)$.

${ }^{9}$ CASULA $1970 \mathrm{n}^{\circ} 2,73,109,112,115,130,158$.

${ }^{10}$ La grande enciclopedia 2007, art. Orvei, Orveis. Sur Ramon de Cardona, voir FERRER I MALLOL s.d..
} 
état, pour 1342, du versement de trois livres d'alfonsins par le recteur de l'ecclesia de Urdofe, Gisarchensis diocesis ${ }^{11}$. Au $\mathrm{XV}^{\mathrm{e}}$ siècle, l'église est plusieurs fois mentionnée, jusqu'à l'intégration de ses revenus à la mense épiscopale de Bisarcio. A cette époque, l'agglomération villageoise, attestée par l'existence d'une paroisse et confirmée par la mention : parrochialis ecclesie sancti Leonardi ville de Ossiri (Osury), semble sinon prospère $\mathrm{du}$ moins assez nombreuse pour que les revenus de la paroisse aient pu constituer une prébende canoniale $^{12}$. Même si les textes ne permettent pas d'attester l'existence d'un village au XIII ${ }^{\mathrm{e}}$ siècle, il est probable que le château a joué un rôle d'attraction d'une population rurale qui s'est maintenue sur place après la destruction de la forteresse. La petite agglomération se maintient en tout cas aux $\mathrm{XIV}^{\mathrm{e}}$ et $\mathrm{XV}^{\mathrm{e}}$ siècles, comme l'atteste sa présence dans la liste des villages compris dans le fief concédé par le souverain aragonais à Giovanni d'Arborea (1339), à Bernardo de Centelles (1421), puis à Francesc Gilabert de Centelles $(1462)^{13}$. Il est sans doute déserté dans la seconde moitié du XV ${ }^{\mathrm{e}}$ siècle. ${ }^{14}$. Par la suite, seule l'église demeure en fonction comme simple église rurale fréquentée une fois par an pour la fête patronale par les habitants d'Ozieri ${ }^{15}$. Une fondation pieuse effectuée en 1504 par Leonardo Hucula d'Ozieri permet d'assurer les revenus nécessaires à son entretien. Elle peut ainsi servir de refuge temporaire, en 1650, à des habitants d'Ozieri fuyant la peste ${ }^{16}$. L'édifice, qui bénéficie d'une description assez minutieuse en 1769 , est en ruine à partir du début du $\mathrm{XIX}^{\mathrm{e}}$ siècle $^{17}$.

L'enquête archéologique a permis de repérer sur place plusieurs zones d'habitat qui ont été reconnues dans une aire d'environ cinq hectares :

- sur un plateau allongé en bordure de la falaise $(170$ x $45 \mathrm{~m})$ portant le nom de « Monte S. Leonardo » : le castrum (zones A et B) ;

- en contrebas de celui-ci, au sud, sur une terrasse inclinée : quartier de constructions rustiques (zone $\mathrm{C}$ ) ;

- à $300 \mathrm{~m}$ au nord, toujours en bordure de la falaise, au voisinage des ruines de l'église (lieudit «S. Leonardo ») : quartier de constructions rustiques (zone D) ;

- au nord-ouest, à 600 m, les ruines d'un bâtiment rectangulaire en pierre portent le toponyme «S. Pietro ».

\footnotetext{
${ }^{11}$ SELLA 1945, n 261. L'identification se base, outre l'appartenance à l'évéché de Bisarcio, sur la comparaison avec les listes de villages du secteur pour 1388 et 1462.

${ }^{12} 1446$ (8 oct.) : SCANO 1940, ${ }^{\circ}$ CIV ; 1448 (21 oct.) : n CXV ; 1466 (10 juil.) : $\mathrm{n}^{\circ}$ CCXXXII. Les variations de graphies du nom sont dues à une confusion, ou une attraction paronymique avec Ozieri, nom de la ville importante la plus proche, dont S. Leonardo d'Urvei constituait une des prébendes canoniales.

${ }^{13}$ La Grande enciclopedia 2007.

${ }^{14}$ La Grande enciclopedia 2007, qui ne cite pas ses sources, indique qu'en 1468 les derniers habitants d'Orveis se sont transférés à Tula (village voisin). Dans sa Corografia (1584), FARA 1835 mentionne comme détruit l'oppidum Orvaei (t. I, p. 68)

${ }^{15}$ Le site est indiqué sur deux cartes générales de la Sardaigne au XVIII ${ }^{\mathrm{e}}$ s. Le symbole figuré qui accompagne le nom (S. Leonardo), est soit une église (Carta del regno di Sardegna delineata nel 1746, éd. PILONI 1974, pl. LXVIII), soit un symbole «terre e luoghi esistenti », selon la légende (Carta della Sardegna, éd. PILONI 1974, pl. LVIII (av. 1763) ; sur une carte régionale contemporaine, c'est un petit bâtiment (Planta del Cabo de Sacer en Sardeña en la qual se ve los estados del ex ${ }^{m o} e^{r}$ duque de Gandia y conde de Oliva mi sen ${ }^{r}$..., Madrid, Archivo Histórico Natiónal, fonds Osuna, reproduite dans DAY 1993, p. 40).

${ }^{16}$ AMADU 1963, p. 169.

17 «(...) l'église, qui n'a pas un mauvais plan, est de style ancien, vaste, divisée en trois nefs par deux rangées de colonnes de bonne pierre, au centre d'une cour à laquelle on accède par une porte, et où existent des habitations en grande partie détruites, mais dont restent encore de l'une d'elles trois pièces entières et habitables, indice de l'existence autrefois d'une population considérable. On y fait la fête deux fois par an (...) », V. Mameli, Relacio historica, Turin, 1769, éd. BUSSA 1985, p. 177-178 (traduction du castillan).
} 
La fouille elle-même a été menée principalement sur le castrum (dans le château et dans six bâtiments), accompagnée de sondages dans les deux autres zones. Elle a permis de distinguer fonctionnellement ces différentes zones d'activités et c'est sur cela que je voudrais insister ici.

Le castrum est établi sur un plateau rocheux couvrant une surface d'environ $3500 \mathrm{~m}^{2}$ et bordé de tous côtés par des falaises abruptes. Cette efficace défense naturelle était peut-être complétée par une enceinte dont les indices ténus subsistent çà et là. De plus, par endroits, les restes infimes d'une base de muraille sont décelables sur le rebord du plateau, mais leur état extrêmement fragmentaire empêche d'affirmer que celle-ci formait une courtine continue, et même d'exclure qu'il ne s'agisse dans plusieurs cas de quelque reste de bâtiment installé au contact de la falaise rocheuse. Au nord, l'accès est aménagé dans une échancrure de la falaise avec une sorte de rampe d'accès présentant par endroits les restes d'un dallage irrégulier de grosses pierres formant plusieurs emmarchements. La table rocheuse comporte dans la partie ouest le château proprement dit, composé d'une aire quadrangulaire de $30 \mathrm{~m}$ de côté fermée par une courtine dont le plan est décelable par la microtopographie de surface, avec un donjon carré dans l'angle sud-est et une autre tour au nord-ouest. Une porte est aménagée au nord, dans la courtine contre la tour du nord-est. L'élément principal, le donjon, est un édifice de 8 $\mathrm{m}$ de côté très soigneusement construit en grand appareil régulier de pierres à bossages (trachyte). Seule la partie inférieure de l'édifice est conservée sur quelques mètres de haut et, à l'extérieur, la récupération des blocs de parement n'a épargné que les trois ou quatre assises inférieures. La comparaison architecturale avec d'autres édifices de même type, comme les châteaux de San Michele (Cagliari), de Barumela (Arborea) ou de Casteldoria (Torres), permet de confirmer une datation au XIII ${ }^{\mathrm{e}}$ siècle $^{18}$.

Dans la partie est du plateau, au-delà d'un espace vide et légèrement déprimé d'une quinzaine de mètres de large environ, se trouve un quartier de bâtiments rustiques. De part et d'autre d'une rue centrale de 3 à $4 \mathrm{~m}$ de large, une dizaine de bâtiments est disposée perpendiculairement à celle-ci, formant dans l'ensemble un plan assez régulier. Leur plan, leur taille et le nombre de leurs pièces est variable mais le mode de construction est identique : ils sont faits de blocs irréguliers de trachyte liés de terre argileuse. Les vestiges de ces bâtiments affleuraient au sol avant la fouille et l'on peut en déduire qu'une bonne partie des couches de destruction des murs a fait l'objet de prélèvements, sans doute pour l'édification de murets de pierres sèches délimitant les zones de pâturage, de sorte que seul l'intérieur des pièces présentait une accumulation pierreuse, rarement très épaisse, et ne permettant en tout cas pas d'estimer la hauteur originelle des murs. Quoi qu'il en soit, le mode de construction de ces murs minces (0,50 à $0,70 \mathrm{~m}$ en moyenne), sans fondations, sans mortier, sans assises et sans calibrage des moellons fait supposer des bâtiments de plain pied et d'élévation très modeste ${ }^{19}$. Examinons rapidement ces bâtiments.

Le bâtiment $\mathrm{V}$, implanté le plus à l'ouest, forme un rectangle allongé de 15 sur $6 \mathrm{~m}$ hors tout (surface intérieure : 42,5 $\mathrm{m}^{2}$ ) avec une porte large de $1,40 \mathrm{~m}$ ouvrant sur la rue dans l'angle nord-est. Une seconde pièce, plus petite $\left(24 \mathrm{~m}^{2}\right)$ est présente au sud, noncommunicante (cette partie du bâtiment est très détruite). À l'intérieur de la pièce la plus grande, on note la présence de deux banquettes basses adossées aux murs longitudinaux, parementées seulement vers l'intérieur de la pièce, de 0,50 à $0,60 \mathrm{~m}$ de large. Ces deux structures ne sont pas contemporaines : on a noté deux niveaux de terre battue, correspondent respectivement à chacune de ces banquettes. Celle de l'ouest est antérieure à celle de l'est, témoignage d'une réfection, sinon de l'ensemble du bâtiment, au moins de la disposition interne. La première phase comportait une banquette à l'ouest, qui fut ensuite arasée, et la deuxième phase, consécutive à un incendie, présente une banquette à l'est.

\footnotetext{
${ }^{18}$ CARRADA et al. 1995, p. 69-101.

${ }^{19}$ POISSON 2002.
} 
La fouille n'a rencontré aucun matériau correspondant à l'effondrement de la toiture, ce qui laisse supposer l'usage de végétaux, et aucun foyer domestique, ce qui pourrait faire écarter la fonction d'habitation pour cette pièce. Le mobilier métallique appartenait principalement à l'équipement du cheval. Il est composé en premier lieu de fers, entiers ou fragmentaires, et clous de fers à cheval, ainsi que diverses boucles, anneaux et passants, que leur matériau, le fer, fait habituellement attribuer au harnachement. Deux objets semblent particulièrement caractéristiques de cette fonction: sur le sol, à proximité d'une des mangeoires a été trouvée une paire d'anneaux en fer de forme quadrangulaire en $\mathrm{T}(10,5 \mathrm{x} 6 \mathrm{x}$ $3,5 \mathrm{~cm}$ ), qui doivent être identifiés comme des dés de sous-ventrière, permettant d'associer une courroie étroite à une courroie large ${ }^{20}$. Des objets de ce type ont été trouvés en contexte médiéval, notamment dans l'aire méditerranéenne, avec des variantes :

- rectangulaires en T (comme à Urvei) à Rome, Crypta Balbi, dans un contexte début XIII et à San Silvestro (Toscane) dans un contexte $2^{\mathrm{e}}$ moitié XIVe ${ }^{21}$;

- trapézoïdales en T à la Crypta Balbi, à Rougiers (Bouches-du-Rhône) dans un contexte du $\mathrm{XIV}^{\mathrm{e}}$, et à Saint-Romain, «Le Verger » (Côte-d'or) ${ }^{22}$;

- en D à Londres, Baynard House, dans un contexte de la $2^{\mathrm{e}}$ moitié du XIV $\mathrm{XI}^{\mathrm{e}}(2 \mathrm{ex} .)^{23}$.

La sangle est évidemment un élément primordial de la fixation de la selle sur le cheval. R. Lefebvre des Noëttes écrit que « à partir de 1130 environ, le cavalier au lieu de sangler son cheval uniquement en arrière des coudes comme de nos jours, le sangle aussi sous le ventre et pousse même la précaution jusqu'à enserrer le tronc de l'animal en un véritable corselet de sangles ${ }^{24}$. Des objets de ce dernier type peuvent avoir servi non seulement au harnachement du cheval de selle, mais aussi, associés à des boucles, au harnais d'attelage, comme le montre l'illustration du dictionnaire de Larive et Fleury $\left(\mathrm{XIX}^{\mathrm{e}} \mathrm{s} \text {. }\right)^{25}$. Il est cependant exclu que des attelages aient pu accéder au plateau.

Ces divers éléments permettent de confirmer l'identification des banquettes comme supports de mangeoires, et de proposer d'attribuer à ce bâtiment la fonction d'une écurie. On peut, d'après le plan de ce bâtiment qui forme un rectangle allongé, restituer une disposition interne où une dizaine de chevaux pouvait être installée, perpendiculairement au long mur supportant la mangeoire, et laissant en arrière de ceux-ci un espace de circulation d'environ $2 \mathrm{~m}$. L’iconographie médiévale nous montre que cette disposition, traditionnelle, était en vigueur au Moyen Âge. Voir notamment l'image illustrant le Livre des merveilles de Marco Polo au fo 92 du manuscrit fr. 2810 de la Bnf (v. 1410) où est représentée une écurie sous la forme d'un bâtiment très allongé, apparemment couvert de chaume, à l'intérieur duquel sont disposés d'un côté mangeoires et râteliers.

Dans l'angle nord-est du bâtiment, près de la porte, contre le mur ouest, une fosse rectangulaire est creusée dans le remblai. Le fond est constitué par la roche en place et une grande dalle la borde vers l'intérieur de la pièce. La présence de traces de feu dans le fond et d'une intense rubéfaction sur le parement du mur à cet endroit a fait proposer de voir dans cet aménagement non pas une petite forge, comme il aurait pu sembler au premier abord, car on n'a pas recueilli de battitures ou autres résidus métalliques, mais peut-être un élément de

\footnotetext{
${ }^{20} \mathrm{Et}$ absolument pas comme des étriers - par leur forme et leurs dimensions (largeur int. : 8,5 cm ; hauteur int. max. : 4,5 cm) - comme cela a été proposé au moment de la restauration des objets, puis dans leur présentation au Musée Sanna de Sassari. Cf. PORRU 2004 (Ch. 3 : Restauro di una staffa di un cavaliere leggero arborense ritrovata nel castello di Urvei). Les objets sont exposés dans la vitrine 67, Cf. ROVINA 2000.

${ }^{21}$ L'esedra della Crypta Balbi 1990, p. 540-541, pl. LXXXI n 714 ; FRANCOVICH et alii 1985, p. 355, pl. II $\mathrm{n}^{\circ} 17$.

${ }^{22}$ L'esedra della Crypta Balbi 1990, pl. LXXXI n 715 ; DEMIANS D’ARCHIMBAUD 1980, p. 483-484, pl. $461 \mathrm{n}^{\circ} 1-2$; GRAPPIN 1979, pl. 8 n 7.

${ }^{23}$ CLARK 1995, p. 60-61, pl. 45 n 49-50 (girth's T-shaped haps).

${ }^{24}$ LEFEBVRE DES NOËTTES 1931, p. 243.

${ }^{25}$ LARIVE et FLEURY 1888, articles « harnais », t. I, p. 938 et « sous-ventrière », t. III, p. 380.
} 
chauffage. L'hypothèse d'une écurie chauffée pourrait paraître fantaisiste, cependant une enquête ethnographique sur place (Chilivani, situé à quelques kilomètres, est un des deux plus grands haras d'Italie) a montré qu'on considère que le mode de couverture en tuiles était préférable au végétal pour les écuries, au motif qu'il maintient mieux la chaleur. Nous sommes dans une zone de montagne où $1^{\prime}$ hiver est froid (isothermes diurnes annuelles de $7^{\circ}$ et $8^{\circ}$ dans ce secteur $^{26}$ ) : peut-être était-il nécessaire, dans une écurie couverte de végétaux, de prévoir pour les chevaux, qui sont sensibles au froid, une source de chaleur en hiver? Remarquons également que sur l'image citée ci-dessus du Livre des merveilles est figurée, à l'extrémité de l'écurie, une petite excroissance maçonnée pourvue d'une cheminée, mais il serait sans doute imprudent d'insister sur cette hypothèse. La pièce sud, dont les murs sont très mal conservés, présentait un sol très grossièrement aménagé entre de nombreux affleurements rocheux : il pouvait s'agir d'une resserre ou d'un fenil.

Le bâtiment VII fait face au précédent, de l'autre côté de la rue, au nord. C'est un rectangle de 19 par $5 \mathrm{~m}$ hors tout (surface intérieure : $67 \mathrm{~m}^{2}$ ). On y a noté également deux phases de construction avec un changement d'emplacement de la porte. Le sol de terre battue ne présentait pas de traces de foyer mais était recouvert par une mince couche de tuiles provenant de l'effondrement du toit. Là également, la présence de mobilier appartenant à l'équipement du cheval, ainsi que le plan en rectangle allongé ont suggéré la fonction d'écurie, malgré les différences architecturales avec le bâtiment V (absence de banquettes et matériau de couverture différent).

Le bâtiment IV comporte deux parties : au nord, un bâtiment de 7 par $6 \mathrm{~m}$ hors tout comprenant deux pièces (surface intérieure : $40 \mathrm{~m}^{2}$ ) et au sud une pièce mitoyenne mais noncommunicante (surface intérieure : $30 \mathrm{~m}^{2}$ ). Au nord, les sols sont composés de terre battue et de nombreux affleurements rocheux. Les aménagements y sont nombreux : restes de dallage de pierres plates, alignements de pierres créant des banquettes légèrement surélevées, rochers aménagés, ainsi que plusieurs zones de foyers à même le sol. La fouille a rencontré à cet endroit de nombreux fragments métalliques ainsi que quelques résidus de fonte de fer. Ces caractéristiques ont fait proposer d'y voir une activité artisanale, sans doute liée au travail du métal (forge ?). La pièce sud comportait un foyer aménagé contre le mur ouest et un sol de terre battue, plan et lisse : là se trouvait sans doute la pièce d'habitation, dotée d'un accès direct à l'extérieur, au sud.

Le bâtiment III comporte une pièce unique au sol de terre battue, avec un foyer construit dans l'angle nord-ouest. Il s'agit d'un aménagement important et soigné, fait d'une sole de briques de terre cuite bordée d'une ligne de pierres. Une paroi l'isole du mur mitoyen à l'ouest. Le caractère imposant de la structure de feu, qui occupe une surface d'environ un huitième de la pièce, ainsi que les nombreuses traces de feu et les restes de faune présents sur le sol, font penser à une cuisine, peut-être à usage collectif (?).

Le bâtiment VI comporte une pièce unique, trapézoïdale, de 6 par $7 \mathrm{~m}$ avec un foyer dans l'angle sud-est. C'est le seul bâtiment fouillé qui semble avoir eu une fonction exclusive d'habitation. Cependant on ne pas préjuger de la fonction des bâtiments non explorés qui sont, dans ce secteur, au nombre d'au moins trois ou quatre à l'est du bâtiment III, un entre les bâtiments VIII et VI et encore peut-être un ou deux à l'est.

En résumé, ce quartier où cinq bâtiments sur huit reconnus de façon certaine, ont été fouillés, semble dévolu à des activités spécialisées. A part les activités militaires, concentrées dans le secteur du château, à proximité immédiate, ce sont les activités liées au cheval, l'artisanat du fer, avec peut-être une forge ou maréchalerie (d'après les analyses de scories, il pourrait s'agir de métallurgie du fer et peut-être du cuivre), ainsi que des dépendances du château (cuisine). L'existence de deux écuries de bonnes dimensions, près du château, est

\footnotetext{
${ }^{26}$ PRACCHI et TERROSU ASOLE (dir.) 1971, pl. 13.
} 
sans nul doute à mettre en relation avec la présence d'une armée composée au moins pour partie de cavalerie, et non pas seulement destinées à abriter les montures d'une petite élite. Cela s'accorde assez bien avec les données textuelles qui semblent assigner au château d'Urvei un rôle essentiellement stratégique et militaire, aussi bien à l'époque de son érection (vers 1250 ?) qu'à celle de sa restauration/réoccupation (vers 1330).

La Sardaigne est une terre de chevaux. Une race autochtone et sauvage existe encore dans le sud-ouest de l'île, le « cavallino della Giara », considéré comme un fossile vivant. Il est petit (1,40 m au garrot) mais ce n'est pas un poney ; c'est un animal rustique, résistant et nerveux, à la robe baie ou brune, la tête carrée sur un cou musclé et la croupe courte. Il est en outre doté d'une crinière abondante et d'un mauvais caractère. On considère que les croisements opérés avec des chevaux arabes au début du Moyen Âge, puis avec des chevaux andalous à la fin du Moyen Âge et à l'époque moderne ont produit le cheval élevé aujourd'hui en Sardaigne et appelé « anglo-arabo-sardo » ${ }^{27}$.

Dès l'Antiquité les chevaux sardes sont utilisés et appréciés : on sait qu'au IV siècle ils étaient importés à Rome pour la remonte de l'armée ${ }^{28}$. L'élevage équin est toujours actif dans la Sardaigne médiévale : dès le XII ${ }^{\mathrm{e}}$ siècle, les actes des cartulaires et les censiers mentionnent de nombreux troupeaux de chevaux sauvages et dressés, ainsi que le personnel chargé des soins aux chevaux, les agasones $^{29}$. Les grands domaines gérés par des établissements ecclésiastiques pisans pratiquent l'élevage équin de façon rationnelle et à grande échelle ${ }^{30}$ Les pactes d'alliance entre les judicats sardes et les puissances étrangères, comme Pise, comportent la fourniture de chevaux pour l'armée ${ }^{31}$. Mais le cheval sarde n'est pas objet de commerce : les Etats s'en réservent le monopole pour leurs troupes de cavaliers ${ }^{32}$, et son exportation est interdite afin de réserver aux garnisons pisanes en poste dans l'île un approvisionnement constant en chevaux. Dans l'acte qui lie Mariano II de Bas, au nom du jeune Niccolo de Capraia, juge d'Arborea, à la Commune de Pise (1250), il est expressément spécifié que les chevaux sont exclus du droit de libre exportation du bétail accordé aux

\footnotetext{
${ }^{27}$ FILIA 1866 ; FIORENTINO 1879.

${ }^{28}$ Ammien Marcellin, Histoire de Rome, XXIX, 3-5 : en 371 Valentinien III fit lapider Constancien préposé aux remontes à qui il avait donné mission d'aller en Sardaigne recevoir des chevaux destinés au service militaire, et qui en avait remplacé quelques-uns de sa propre autorité. Quelques témoignages iconographiques de l'élevage équin en Sardaigne dans le haut Moyen Âge sont fournis par des représentations de chevaux marqués : d'un chrisme sur la cuisse gauche (épitaphe de l'aurige (?) Karissimus sur la mensa funéraire de Tharros, IV ${ }^{\mathrm{e}} \mathrm{s}$. (DUVAL 1982, p. 281-285), d'une croix sur la cuisse droite et un triangle sur l'épaule droite sur la plaque de bronze de Sant'Antioco, $\mathrm{X}^{\mathrm{e}}-\mathrm{XI}^{\mathrm{e}}$ s. (SPANO 1864, p. 49-51).

${ }^{29}$ Vers 1120 un noble sarde Furato de Gitil et sa femme Susanna de Thori donnent au monastère S. Niccolò de Soliu un troupeau de 50 juments et 20 chevaux domestiqués (caballos domatos) : SABA 1927, $\mathrm{n}^{\circ} 16$; en 1153 le juge de Torres Gonnario de Lacon donne au monastère cassinien de S. Maria de Thergu un grand domaine de saltus pour que les moines y fassent paître leurs troupeaux équins (pro caballos et pro ebbas): SABA $1927 \mathrm{n}^{\circ}$ 31 ; la dotation du monastère cistercien de S. Maria de Paulis, lors de sa fondation en 1205, comprend des troupeaux considérables de bovins, ovins et porcins, et en particulier 200 juments et 100 chevaux (caballos centum inter domitos et domandos) :TOLA 1861, XIIIe s., $\mathrm{n}^{\circ} 5$.

${ }^{30}$ L'inventaire des biens de l'Euvre de la cathédrale de Pise au domaine d'Astia (judicat de Cagliari) en 1270 mentionne 2 chevaux (equos gualagnos: étalons ?), 37 juments, 7 poulains à dresser (pultros a domando) et 5 poulains de l'année (Archivio di Stato de Pise, fonds Primaziale, n 439 (17 oct.) ; en 1272 : 2 chevaux (equos massarghios : étalons ?), 39 juments poulinières (iumenta de matre), 9 poulains, 10 poulains de l'année et 6 pouliches de l'année ( $\mathrm{n}^{\circ} 440$ (11 mars). BAUTIER 1978, p. 50-54.

${ }^{31}$ Le code rural promulgué par Mariano IV d'Arborea prévoit des dispositions spéciales pour l'élevage équin (inséré dans la Carta de Logu, ch. CLIII, où sept articles concernent les chevaux), Cf. FOIS 1990, p. 165-198.

${ }^{32}$ Les pactes signés entre Pietro juge de Cagliari et la commune de Gênes mentionnent la promesse de fournir des chevaux pour l'armée : en 1176, et dabo militibus equos (TOLA 1861, XIIe s., $\mathrm{n}^{\circ} 107$ ); et en 1186, equos universis militibus de exercitu eorum dabo (...) equos sufficienter dabo ( $\left.{ }^{\circ} 119\right)$.
} 
marchands pisans ${ }^{33}$. On rappellera que ce juge d'Arborea et présumé avoir édifié le château d'Urvei.

Lors de la visite pastorale qu'il effectue en Sardaigne en 1263, l'archevêque de Pise Federico Visconti reçoit en cadeau du juge d'Arborea un superbe cheval blanc, qui constitue à la fois l'emblème du prestige du prélat (les archevêques de Pise ont obtenu en 1118 l'usage du cheval blanc ${ }^{34}$ ), et celui de la qualité de l'élevage sarde ${ }^{35}$. Dans les inventaires et les censiers pisans du XIVe siècle sont mentionnés des pâturages pour les chevaux, ainsi que des bâtiments pour la paille et les chevaux ${ }^{36}$. Après la conquête aragonaise, l'élevage équin se développe encore et permet des exportations massives vers la péninsule Ibérique ${ }^{37}$.

On le voit, l'élevage équin est en Sardaigne non seulement un secteur important de l'économie d'élevage, qui concerne aussi les ovins, mais avec une finalité différente. C'est aussi une production stratégique dans un territoire en guerre presque permanente au cours des XIIIe et XIVe siècles des pouvoirs locaux entre eux d'une part, et avec les puissances coloniales d'autre part.

A Urvei, il semble donc bien que ce qui caractérise les structures édifiées sur la plateforme du castrum est leur statut d'éléments complémentaires du château, de dépendances placées à proximité immédiate de lui et protégées par sa présence, qui confère à ce quartier « spécialisé » une fonction de basse-cour. La mise au jour dans ce secteur de mobilier d'un type particulier, les céramiques d'importation toscane ou ligure, comme les maioliche arcaiche et les types à sgraffito tirrenico viennent aussi à l'appui de cette constatation. La présence de quelques habitations n'est pas incompatible avec cette fonctionnalité : il peut s'agir de dépendants qui résident au plus près de leurs activités d'artisans ou de serviteurs, si l'on considère que les soldats eux-mêmes sont logés dans les bâtiments du château proprement dit. Il faut donc sans doute chercher ailleurs la communauté d'habitants villageois attestée sur le site, et examiner la situation dans le secteurs extérieur au plateau.

Sur un replat en contrebas du castrum au sud (secteur C), est installé un quartier d'habitat hors les murs. Sur les six bâtiments reconnus dans cette zone, un seul a été fouillé. Il s'agit d'un bâtiment quadrangulaire qui n'a pas été totalement reconnu au sud (ou devait se trouver l'accès). Sa largeur est de $6,50 \mathrm{~m}$ et sa longueur de plus de $6,00 \mathrm{~m}$, soit une taille plus grande que les maisons du castrum. Les murs sont faits là aussi de blocs irréguliers de trachyte local liés d'argile. Le bâtiment est séparé de ses voisins, à l'ouest et à l'est, par une ruelle. Au nord, un espace rectangulaire de 9 x 6,50 m est contigu et accolé au bâtiment, sans communication avec lui. Cet espace s'ouvre à l'est par une étroite porte $(0,80 \mathrm{~m})$ dans l'angle sud-est. La stratigraphie s'est révélée très différente dans les deux parties, de part et d'autre du mur ouest-est : au sud, une couche de destruction assez importante recouvrait une couche de tuiles et au-dessous un sol de terre battue assez soigneusement aménagé. Au nord, on n'a pas constaté d'accumulation de pierres, ni de couche de tuiles mais, à peine sous la surface, un niveau d'occupation très irrégulier et en assez fort pendage nord-sud, comprenant beaucoup de restes. Deux foyers aménagés ont été rencontrés, composés d'une sole de terre durcie par l'action du feu entourée d'une bordure circulaire de pierres, de part et d'autre du mur estouest, approximativement au milieu. Des comparaisons ethnographiques permettent

\footnotetext{
${ }^{33}$ Ita quod omnes et singuli Pisani, et qui Pisano nomine censentur, possint mittere et extrahere bestias vivas et mortuas, exceptis equis, in villa et de villa Arestani, sine aliquo dirictu (BONAINI 1854, p. 603).

${ }^{34}$ KEHR 1908, III, p. 321, n 12.

${ }^{35}$ Unum pulcrum pallafridum album cum noblissima sella sardica multarum corrigearum valente libris XV, Cf. BERIOU 2001, p. 1067.

${ }^{36}$ ARTIZZU 1961, p. 215-299.

${ }^{37}$ Mentions d'exportations : juments d'Alghero vers Majorque, de roncins vers Barcelone et Marseille (141011). TODDE 1959 ; GUERRA s.d.
} 
d'envisager des fours culinaires dont la voûte s'est effondrée. Il apparaît donc que l'on a affaire au sud à une maison d'habitation couverte de tuiles et au nord à un espace découvert, sans doute une cour, peut-être destinée à parquer des ovins (surface : 58,5 $\mathrm{m}^{2}$ ). La présence d'un four extérieur est également attestée par l'observation ethnographique, avec notamment le mode de cuisson traditionnel du pain : des galettes (focacce) appelées pane succenericcio (« cuit sous la cendre ») ou ses variantes locales ${ }^{38}$.

Même si l'exploration de ce secteur n'a pas été très étendue, on a bien l'impression qu'il s'agit là d'un véritable quartier d'habitations rustiques où devait habiter la population rurale permanente, avec des modes de vie et des activités différentes des habitants du castrum, sans doute d'effectifs variables. Ici ce sont des bergers et des paysans, les paroissiens de l'église San Leonardo voisine, près de laquelle se trouvait aussi une autre zone d'habitat, mal reconnue.

La description des différentes structures fait apparaître une différence relativement nette, sinon dans les modes de construction, mais dans les modes d'occupation et l'organisation fonctionnelle, entre la zone du castrum proprement dit et les zones « villageoises ». Sur le plateau, au contact du château, sont localisés des bâtiments étroitement associés fonctionnellement à celui-ci (écuries, bâtiments artisanaux, dépendances). Quant à la population rurale - qui reste notablement dispersée par rapport au pôle agglomérant du château - elle est installée dans des sites nettement distincts de la zone castrale. Il faut alors se rappeler que nous sommes ici dans un contexte colonial et il est possible que, à l'instar des villes où le fait est attesté par la réglementation, la population sarde n'a pas le droit de demeurer dans les fondations, pisanes notamment ${ }^{39}$.

Le château d'Urvei semble avoir connu une fin brutale, d'après l'interprétation des sources textuelles, dans les premières décennies du XIV ${ }^{\mathrm{e}}$ siècle, et les éléments de datation fournis par la fouille semblent confirmer cette appréciation. A part un abondant mobilier antique (céramique, verre, monnaies, fragments architecturaux) qui atteste une occupation aux premiers siècles ap. J.C., sur le site ou à proximité immédiate, qui n'est pas en place mais présent seulement dans les couches de fondation ou de remblais, l'occupation du site est assurée à l'époque médiévale. Les éléments les plus anciens sont des fragments de céramique du type sparse glazed ware $\left(\mathrm{XII}^{\mathrm{e}}-\mathrm{XIII}^{\mathrm{e}} \mathrm{s}\right.$.), mais la plupart du mobilier associé aux couches d'occupation et de destruction est constitué par des céramiques du type maiolica arcaica d'origine pisane et sgraffito tirrenico d'origine génoise (fin XIII ${ }^{\mathrm{e}}$ - début $\mathrm{XIV}^{\mathrm{e}}$ s.). S'ajoutent à cela cinq monnaies, elles aussi de provenances pisane et génoise :

+ . FR . IM / PATOR $\because$, aigle à gauche, sur un chapiteau ; PI / SE (une étoile $\star$ au-dessus du $\mathrm{P})$, Vierge à l'enfant dont la tête est encadrée par les lettres $\Pi P / \Theta Y$

République de Pise, demi-gros au nom de Frédéric Ier empereur (1296-1312), $\operatorname{argent}^{40} \quad$ : b. V (occupation) ; b. VIII (sol); b. III (sol);

+ CVNRADI REX, croix pattée ; + I . A . N . V . A ., château (porte)

République de Gênes, denier au nom de Conrad (1138-1339), $\operatorname{argent}^{41}$ : b. III (sol) ; b. VII (incendie).

Ainsi que des datations au radiocarbone :

- b. VIII (destruction) : 865-1020 ;

- b. V (incendie) : 1190-1295 ; b. VII (sol) : 995-1260

- B donjon (incendie) : 1225-1325 ; b. V (sol supérieur) : 1260-1335

\footnotetext{
${ }^{38}$ BALDACCI 1952, p. 109 ; COSSU 1894, p. 320-321.

${ }^{39}$ Cf. POISSON 1995, p. 39-49.

${ }^{40}$ Corpus Nummorum Italicarum 1911, II, n 63 ; BALDASSARI (dir.), 2010.

${ }^{41}$ GRIERSON et TRAVAINI 1998.
} 
Ces éléments font proposer trois phases d'occupation médiévale : $\mathrm{XI}^{\mathrm{e}}$ siècle, $\mathrm{XIII}^{\mathrm{e}}$ siècle et début $\mathrm{du} \mathrm{XIV}^{\mathrm{e}}$ siècle, qui sont compatibles avec la chronologie proposée par les données historiques.

Pour finir, il semble intéressant de noter, avec ce modeste exemple méditerranéen, l'importance accordée au cheval dans un site castral assez rustique, mal adapté a priori à l'utilisation et au logement d'un tel animal. La présence de chevaux dans les châteaux est certes attestée ailleurs en Sardaigne: au $\mathrm{XV}^{\mathrm{e}}$ siècle, le palefrenier du comte Berengario Carroz au château de San Michele (au nord de Cagliari) était chargé de prendre un soin particulier de ses deux destriers «Thomas » et «Benedito », ainsi que de la pouliche noire de sa fille Violante, appelée «Puça ${ }^{43}$. Ce château est cependant d'un accès beaucoup plus facile qu'Urvei, et l'attestation archéologique d'écuries dans un site castral de montagne ne peut que confirmer la place capitale du cheval dans l'activité militaire médiévale, composée le plus souvent de « chevauchées » à partir des châteaux.

\section{$\underline{\text { Sources }}$}

\section{ARTIZZU F.}

1961, «Liber fondachi », Annali della Facoltà di Lettere e Filologia dell'Università di Cagliari 29, 1961-65, p. 215-299.

BERIOU N.

2001, Les sermons et la visite pastorale de Federico Visconti archevêque de Pise (1253-1277), Rome, Ecole française de Rome.

BONAINI F.

1854, Statuti inediti della città di Pisa, dal XII al XIV sec., Florence, t. I.

BONAZZI G.

1900, Il condaghe di San Pietro di Silki, Sassari, réed. 1979.

CASULA F.C.

1970, Carte reali diplomatiche di Alfonso III il Benigno re d'Aragona, riguardanti l'Italia, Padoue.

FOIS B.

1990, Territorio e paesaggio agrario nella Sardegna medievale, Pise, p. 165-198.

KEHR P.F.

1908, Italia pontificia, Berlin, Regesta Pontificum Romanorum, III.

SABA A.

1927, Montecassino e la Sardegna medievale. Codice diplomatico sardo-cassinese, Mont-Cassin.

SALAVERT Y ROCA V.

1956, Cerdeña y la expansión mediterránea de la Corona de Aragón (1297-1314), Madrid, vol. II, Documentos. SCANO D.

1940, Codice diplomatico delle relazioni tra la Santa sede e la Sardegna, Cagliari.

SELLA P.

1945, Sardinia. Rationes decimarum Italiae (XII-XIV sec.), Vatican, Studi e Testi 113.

TOLA P.

1861, Codex diplomaticus Sardiniae, Turin, réed. Sassari, 1994.

\section{Bibliographie}

\section{AMADU F.}

1963, La diocesi medievale di Bisarcio, Cagliari, réed. 2003.

1975, «Castelli e ville medievali del Logudoro e del Goceano », Archivio storico sardo di Sassari 1, réédité dans ID., La diocesi medievale di Castro, Ozieri, 1984, p. 173-214.

1978, Ozieri e il suo territorio dal neolitoco all'età romana, Cagliari.

BALDACCI O.

1952, La casa rurale in Sardegna, Florence, Riocerche sulle dimore rurali in Italia VIII.

\footnotetext{
${ }^{42}$ Archéolabs, ARC 1213, 1206, 1212, 1208, 1205.

${ }^{43}$ FERRANTE 1992, p. 139.
} 
BALDASSARI M. (dir.)

2010, Zecca e monete del Comune di Pisa, vol. I, Dalle origini agli inizi della Seconda Repubblica, XII sec. 1406, Pise.

BAUTIER R. et A.-M.

1978, « Contribution à l'histoire du cheval au Moyen Âge », Bulletin philologique et historique du C.T.H.S., p. 9-75 (« Le cheval en Italie », p. 50-54).

BUSSA I.

1985, Quaderni bolotanesi 11, p. 177

CARRADA F. et al.

1995, «L'uso del "bugnato" nella Sardegna medievale», Materiali per una topografia urbana, Oristano (Mediterraneo tardoantico e medievale 10), p. 69-101.

CLARK J.

1995, The medieval horse and its equipment, c. 1150 - c. 1450, Londres, Medieval finds from excavations in London 5.

Corpus Nummorum Italicorum

1911, Rome, t. II (Piemonte -Sardegna).

COSSU M.

1894, « Cibi tradizionali », Rivista delle tradizioni popolari italiane I, fasc. IV, p. 320-322.

DAY J.

1973, Villaggi abbandonati in Sardegna dal trecento al settecento : inventario, Paris, IRHT.

1993, Atlas de la Sardaigne rurale aux $17 e ̀$ et $18^{e}$ siècles, Paris, EHESS.

DEMIANS D'ARCHIMBAUD G.

1980, Les fouilles de Rougiers, Paris.

DUVAL N.

1982, «Une mensa funéraire de Tharros (Sardaigne) et la collection chrétienne du Musée de Cagliari », Revue des études augustiniennes XXVIII, p. 280-288.

L'esedra della Crypta Balbi nel medioevo (XI-XV s.)

1990, Florence, Biblioteca di Archeologia Medievale, Archeologia urbana a Roma : il progetto della Crypta Balbi 5.

FARA G.B.

1835, De corographia Sardiniae (1584), éd. A. Cibrario, Turin, réed. E. Cadoni Sassari, 1992.

FERRANTE C.

1992, « La vita sociale nei castelli sardi nell'età aragonese (secc. XIV-XV) », Archivio storico sardo XXXVII, p. 125-143.

FERRER I MALLOL M.T.

s.d., «La circulation des élites militaires: les chefs de guerre catalans en Italie au XIVe siècle», El Mediterraneo medieval desde el observatorio de la Corona de Aragón. Conflictividad e intercambios económicos (PB97-1145), Barcelone, digital CSIC.

FILIA M.

1866, Relazione sulle razze dei cavalli sardi, Salerne.

FIORENTINO V.

1879, Il cavallo sardo, Naples.

FRANCOVICH R. et alii

1985, «Un villaggio di minatori e fonditori di metallo nella Toscana del Medioevo : San Silvestro (Campiglia marittima) », Archeologia medievale XII, p. 313-401.

La grande enciclopedia della Sardegna

2007, Sassari, vol. 7 (Orticoltura - Quasina).

GRAPPIN S.

1979, Les fouilles1979 au lieu-dit «Le vieux château », Saint-Romain (Côte-d'or). Rapport de fouilles $n^{\circ} 6$ (dactylographié).

GRIERSON P. et TRAVAINI L.

1998, Medieval european coinage, Cambridge.

GUERRA U.

s.d., Antologia di Sardegnacavalli.it. Evoluzione equestre, www.sardegnacavalli.it

LA CORTE G.

1899, La scolca e il suo maiore. I buiakesos, Sassari.

LARIVE et FLEURY

1888, Dictionnaire français illustré des mots et des choses, Paris, 3 vol.

LEFEBVRE DES NOËTTES R.

1931, L'attelage. Le cheval de selle à travers les âges, Paris. 
MELONI G.

1988, « Il Monte Acuto nel medioevo », Mediterraneo e Sardegna nel Basso Medioevo, Cagliari, p. 29-67.

1991, «Insediamento rurale nella Sardegna settentrionale. Tula e il suo territorio nel medioevo », Medioevo saggi e rassegne 15, p. 21-53.

1992, « Il castello di Orvei », dans F. FOIS, Castelli della Sardegna medievale, Milan, p. 227-229.

1992, « Il castello di Monte Acuto », Archivio storico sardo XXXVII, p. 89-124, réed. Ozieri. 1994.

PILONI L.

1974, Carte geografiche della Sardegna, Cagliari, réed. 1997.

POISSON J.-M.

1986, «Prospections archéologiques en Sardaigne», Histoire et archéologie de l’habitat médiéval, Lyon, CIHAM.

1990, "Castelli medievali di Sardegna : dati storici e dati archeologici", Lo scavo archeologico di Montarrenti e $i$ problemi dell'incastellamento medievale (colloque de Sienne, déc. 1989), Archeologia medievale XVI, p. 191204.

1991, « Ozieri (Sassari). Località San Leonardo. Prima campagna di ricerche archeologiche nel sito di Urvei », Bollettino di Archeologia 10, 1991 (1993)

1992, « Ozieri (Sassari). Urvei. Località San Leonardo. Seconda campagna di scavi », Bollettino di Archeologia 13-15, 1992 (1994).

1995, "Formes urbaines de la colonisation pisane en Sardaigne", dans BALARD M. et DUCELIER A., Coloniser au Moyen Age, Paris, p. 39-49.

2002, «La maison rurale médiévale en Sardaigne : un atelier d'ethnoarchéologie », Ruralia IV. The rural house from the migration period to the oldest still standing buildings, Prague.

PORRU L.

2004, La conquista della Sardegna da parte dell'infante Alfonso vista dalle cronache del tempo, mémoire de laurea, Université de Sassari.

PRACCHI R. et TERROSU ASOLE A. (dir.)

1971, Atlante della Sardegna, fasc. I, Cagliari.

RASI P.

1937, «Exercitus Italicus » e milizie nell'Alto Medioevo, Padoue.

ROVINA D.

2000, La sezione medievale del Museo G.A. Sanna di Sassari.

SPANO G.

1864, « Oggetti figurati e simboli cristiani », Bulletino archeologico sardo X, $\mathrm{n}^{\circ} 5$.

TODDE G.

1959, « L'esportazione dei cavalli dalla Sardegna nel sec. XV », Atti del VI ${ }^{\circ}$ congresso de Historia de la Corona de Aragon, Madrid.

Mots-clés : Sardaigne - château - écuries - Moyen-Âge

\section{Résumé}

Les fouilles archéologiques menées sur le site d'Urvei (prov. Sassari, Italie) ont permis l'étude d'un ensemble castral édifié au moment de la domination pisane sur la Sardaigne (XIIIe -XIVe s.) pour contrôler les territoires septentrionaux face à la présence génoise. Implanté sur une plateforme rocheuse dans une position dominante, le site comportait deux ensembles distincts : le château proprement dit autour d'un fort donjon carré, et un ensemble de dépendances comprenant entre autres une cuisine et deux écuries. Ces dernières, par leur architecture, leurs aménagements et le mobilier qui y a été trouvé, permettent de documenter les caractères de la cavalerie pisane au moment de la probable destruction militaire du château dans la seconde moitié du XIVe siècle.

\section{Summary}

The archaeological excavations on the Urvei site (Sassari province, Italy) have allowed the 
study of a castle ensemble built at the time of the domination of Pisa over Sardinia (13th to 15 th century) in order to control the northern territories facing the Genoese presence. Set on a rocky platform in a dominant position, the site was made of two distinct buildings: a castle in itself around a strong square dungeon and a set of outbuildings among which a kitchen and two stables. These last two buildings, given their architecture, their interior designs and the furniture that was found, allow us to attest the characteristics of the Pisan cavalry at the time of the probable military destruction in the second half of the 14th century.

\section{Légendes des illustrations}

Fig. 1. Carte de la Sardaigne : les influences politiques au XIVe siècle (élab. J.-M. P. d'après F.C. Casula)

Fig. 2. Plan du site d'Urvei.

Fig. 3. Le bâtiment V (écurie), du nord.

Fig. 4. Schéma de coupe en travers du bâtiment V.

Fig. 5. Eléments de harnachement in situ dans le bâtiment V.

Fig. 6. Plan du bâtiment VIII (écurie).

Fig. 7. Objets de harnachement.

Fig. 8. Tableaux de comptage des objets.

Fig. 9. Plan du bâtiment III (cuisine ?).

Fig. 10. Plan du bâtiment XII (maison). 